\title{
Binary nucleation beyond capillarity approximation
}

\author{
V. I. Kalikmanov* \\ Twister Supersonic Gas Solutions, Einsteinlaan 10, 2289 CC Rijswijk, The Netherlands \\ and Department of Geosciences, Delft University of Technology, Stevinweg 1, 2628 CN Delft, The Netherlands \\ (Received 11 March 2010; revised manuscript received 26 March 2010; published 11 May 2010)

\begin{abstract}
Large discrepancies between binary classical nucleation theory (BCNT) and experiments result from adsorption effects and inability of BCNT, based on the phenomenological capillarity approximation, to treat small clusters. We propose a model aimed at eliminating both of these deficiencies. Adsorption is taken into account within Gibbsian approximation. Binary clusters are treated by means of statistical-mechanical considerations: tracing out the molecular degrees of freedom of the more volatile component, we obtain a coarse-grained system described in terms of the single-component mean-field kinetic nucleation theory [V. I. Kalikmanov, J. Chem. Phys. 124, 124505 (2006)], allowing an adequate treatment of clusters of arbitrary size. The model opens a route toward studying binary nucleation in complex systems with nanosized critical clusters.
\end{abstract}

DOI: 10.1103/PhysRevE.81.050601

PACS number(s): 64.60.Q-, 05.20.Jj, 82.60.Nh

Binary nucleation plays a major role in a large variety of physical processes ranging from atmospheric science to materials science. Up to date the main theoretical tool describing this phenomenon is the binary classical nucleation theory (BCNT) pioneered by Reiss in 1950 [1] and developed by Stauffer [2] and based on the capillarity approximation. The latter considers a cluster to be an object with a rigid boundary and uniform intensive properties of the bulk liquid inside it. It has been known for quite a while that predictions of BCNT can differ by many orders of magnitude from the experimental data $[3,4]$ and even lead to unphysical results violating the nucleation theorem [5]. One of the explanations of this discrepancy is the inability of BCNT to take into account the adsorption effects. Wilemski [6] proposed the revised BCNT based on the combination of capillarity approximation with the Gibbs concept of dividing surface. For a binary (and, more generally, a multicomponent) system the Gibbs construction is not just a useful tool (as in the singlecomponent case) but a necessity since it is impossible to choose a dividing surface for a mixture in such a way that adsorption terms for all species vanish [7].

Taking into account adsorption within the phenomenological approach does not resolve another deficiency of the capillarity approximation: the latter assumes that the surface energy of a cluster can be described in terms of the plain layer surface tension. Obviously, for small clusters this concept looses its meaning. This difficulty is not unique for the binary problem-it appears also in the single-component case. In a recently formulated mean-field kinetic nucleation theory (MKNT) [8] this problem was tackled by formulating an interpolative model between small clusters treated using statistical-mechanical considerations and big clusters described by the capillarity approximation. This approach proved to be successful in predicting nucleation in various microscopically diverse substances at conditions when critical clusters are nanosized objects $[8,9]$. Statisticalmechanical treatment of binary clusters in the present model originates from the analogy with the soft condensed-matter

\footnotetext{
*vitaly.kalikmanov@twisterbv.com
}

theory where the description of complex fluids can be substantially simplified if one eliminates the degrees of freedom of solvent molecules to be left with the system of solute particles with an effective Hamiltonian [10]. The situation in nucleation theory is somewhat similar: the complexity of binary problem can be substantially reduced if, considering an arbitrary cluster, we trace out the molecular degrees of freedom of the more volatile component. The resulting pseudoone-component cluster can be then treated by means of MKNT.

Following classical theory, we assume that the elementary process which changes the size of a cluster, containing $n_{a}$ molecules of component $a$ and $n_{b}$ molecules of component $b$, is the attachment to it or loss by it of one molecule, either $a$ or $b$. These processes are characterized by the impingement and evaporation rates per unit surface, $\nu_{i}$ and $\beta_{i}$, where $i$ $=a, b$. For vapor to liquid nucleation $\nu_{i}$ is given by gas kinetics $\nu_{i}=y_{i} p^{\mathrm{v}} / \sqrt{2 \pi m_{i} k_{\mathrm{B}} T}$, where $p^{\mathrm{v}}$ is the total pressure, $T$ is the temperature, $k_{\mathrm{B}}$ is the Boltzmann constant, $m_{i}$ is the mass of a molecule of component $i$, and $y_{i}$ is its molar fraction in the vapor; $\beta_{i}$ are obtained from the detailed balance condition stating that in equilibrium net fluxes along $n_{a}$ and $n_{b}$ vanish. In BCNT it is applied to the constrained equilibrium state which would exist for the vapor at the same temperature, pressure, and vapor phase activities as the vapor in question. Instead of using this artificial state we use the full thermodynamic equilibrium of the system " $a+b+$ carrier gas" at $p^{\mathrm{v}}$ and $T$. This procedure is a natural extension to binary mixtures of the Katz kinetic approach [11]. The quantities referring to full equilibrium are denoted by the subscript "eq." We choose this state as a reference for the vapor phase activities $\mathcal{A}_{i}^{\mathrm{v}} \approx S_{i}=y_{i} p^{\mathrm{v}} /\left[y_{i, \mathrm{eq}}\left(p^{\mathrm{v}}, T\right) p^{\mathrm{v}}\right]=y_{i} / y_{i, \mathrm{eq}}$. Within the standard rate theory, combined with the detailed balance at full equilibrium, the nucleation flux is

$$
\mathbf{J}=-\left[\varrho_{\mathrm{eq}}(\mathbf{n}) \prod_{i=a, b} S_{i}^{n_{i}}\right] \Re \nabla H, \quad H=\frac{\varrho(\mathbf{n})}{\varrho_{\mathrm{eq}}(\mathbf{n}) \prod_{i} S_{i}^{n_{i}}},
$$

where $\varrho(\mathbf{n})$ is the cluster distribution function, $\mathbf{n}=\left(n_{a}, n_{b}\right)$, $\nabla \equiv\left(\partial / \partial n_{a}, \partial / \partial n_{b}\right)$, and the diagonal matrix $\mathfrak{R}$ contains the 
rate of monomer collisions with the cluster surface $A(\mathbf{n})$ : $\mathfrak{R}_{11}=\nu_{a} A(\mathbf{n}), \mathfrak{R}_{22}=\nu_{b} A(\mathbf{n}), \mathfrak{R}_{12}=\mathfrak{R}_{21}=0$. A general form of $\varrho_{\text {eq }}(\mathbf{n})$ is

$$
\varrho_{\mathrm{eq}}\left(n_{a}, n_{b}\right)=C e^{-g_{\mathrm{eq}}\left(n_{a}, n_{b}\right)},
$$

where $g_{\text {eq }}=\beta \Delta G_{\text {eq }}, \beta=1 / k_{\mathrm{B}} T, \Delta G_{\mathrm{eq}}\left(n_{a}, n_{b}\right)$ is the Gibbs free energy of cluster formation, and $C$ is the normalization constant. Equation (1) is similar to the one discussed in BCNT in which the constrained equilibrium distribution is replaced with the product of $\varrho_{\text {eq }}$ and nonequilibrium terms. According to [1] the saddle point $\mathbf{n}^{*}=\left(n_{a}^{*}, n_{b}^{*}\right)$ of the free-energy surface,

$$
g\left(n_{a}, n_{b}\right)=-\sum_{i=a, b} n_{i} \ln S_{i}+g_{\text {eq }}\left(n_{a}, n_{b}\right),
$$

corresponds to the critical cluster. Applying the standard arguments [2] to Eqs. (1) and (2) we find for the total steadystate nucleation rate

$$
J=\nu_{\mathrm{av}} A^{*} \mathcal{Z}\left(\prod_{i} S_{i}^{n_{i}^{*}}\right) \varrho_{\mathrm{eq}}\left(n_{a}^{*}, n_{b}^{*}\right),
$$

where $\nu_{\mathrm{av}}(\varphi)=\nu_{a} \nu_{b} /\left(\nu_{a} \sin ^{2} \varphi+\nu_{b} \cos ^{2} \varphi\right)$ is the average impingement rate, $\varphi$ is the flow direction at the saddle point, $A^{*}=A\left(n_{a}^{*}, n_{b}^{*}\right)$, and $\mathcal{Z}$ is the Zeldovich factor. Equation (3) contains the yet undetermined angle $\varphi$, which is found by maximizing the angle-dependent part of $J$. The advantage of Eq. (3) is that it reduces the binary nucleation problem to the determination of $\varrho_{\text {eq }}\left(n_{a}, n_{b}\right)$.

Within Gibbsian approximation one distinguishes between the bulk (interior) molecules in the cluster (superscript "l") and the excess molecules (superscript "exc") accumulated on the infinitely thin dividing surface: $n_{i}=n_{i}^{1}+n_{i}^{\mathrm{exc}}$. Both $n_{i}^{1}$ and $n_{i}^{\text {exc }}$ depend on the location of the dividing surface, while their sum can be assumed independent of it to the relative accuracy of $O\left(\rho^{\mathrm{v}} / \rho^{1}\right)$, where $\rho^{\mathrm{v}}$ and $\rho^{1}$ are the number densities in the vapor and liquid phases, respectively. A special choice, adopted in the present model, is the equimolar surface for the mixture, or the $K$ surface, introduced by Laaksonen et al. [12], defined through the requirement $\sum_{i} n_{i}^{\mathrm{exc}} v_{i}^{1}=0$, where $v_{i}^{1}$ is the partial molecular volume of component $i$ in the liquid phase. The combination of this expression with the Gibbs adsorption equation results in the identification of $n_{i}^{\text {exc }}$ [13]. This choice ensures that the macroscopic surface tension is independent of the cluster curvature [5].

The partition function of an arbitrary cluster is

$$
Z_{n_{a} n_{b}} \equiv Z_{\mathbf{n}}=\frac{1}{\Lambda_{a}^{3 n_{a}} \Lambda_{b}^{3 n_{b}}} q_{n_{a} n_{b}},
$$

where $\Lambda_{i}$ is the thermal de Broglie wavelength of a molecule of component $i$, and $q_{n_{a} n_{b}}$ is the configuration integral of the cluster in a domain of volume $V$,

$$
q_{n_{a} n_{b}}(T)=q_{\mathbf{n}}(T)=\frac{1}{n_{a} ! n_{b} !} \oint_{\mathrm{cl}} d \mathbf{R}^{n_{a}} d \mathbf{r}^{n_{b}} e^{-\beta U_{\mathbf{n}}},
$$

where $\mathbf{R}^{n_{a}}$ and $\mathbf{r}^{n_{b}}$ are locations of molecules $a$ and $b$ in the cluster, and $U_{\mathbf{n}}=U_{a a}\left(\mathbf{R}^{n_{a}}\right)+U_{b b}\left(\mathbf{r}^{n_{b}}\right)+U_{a b}\left(\mathbf{R}^{n_{a}}, \mathbf{r}^{n_{b}}\right)$ is the potential interaction energy in the cluster. The symbol $\oint_{\mathrm{cl}}$ denotes that integration is only over those molecular configu- rations that belong to the cluster. We represent the equilibrium gaseous state of the $a-b$ mixture as a system of noninteracting $\mathbf{n}$ clusters. The partition function of the gas of $N_{\mathbf{n}}$ of such clusters is factorized: $Z^{(\mathbf{n})}=\left(1 / N_{\mathbf{n}} !\right) Z_{\mathbf{n}}^{N_{\mathbf{n}}}$. The chemical potential of a cluster in this gas using Stirling's formula and Eq. (4) reads

$$
\mu_{\mathbf{n}}=k_{\mathrm{B}} T \ln \left[\varrho_{\mathrm{eq}}\left(n_{a}, n_{b}\right) \frac{V \Lambda_{a}^{3 n_{a}} \Lambda_{b}^{3 n_{b}}}{q_{\mathbf{n}}}\right],
$$

where $\varrho_{\text {eq }}\left(n_{a}, n_{b}\right)=N_{\mathbf{n}} / V$. Equilibrium between the cluster and surrounding vapor requires $\mu_{\mathbf{n}}=n_{a} \mu_{a, \text { eq }}^{\mathrm{v}}+n_{b} \mu_{b \text {,eq }}^{\mathrm{v}}$, where $\mu_{i, \mathrm{eq}}^{\mathrm{v}}\left(p^{\mathrm{v}}, T\right)$ is the chemical potential of a molecule of component $i$ in the equilibrium vapor. Then using Eq. (5), we find

$$
\varrho_{\mathrm{eq}}\left(n_{a}, n_{b}\right)=\left(\frac{q_{\mathbf{n}}}{V}\right)\left[z_{a, \mathrm{eq}}\right]^{n} a\left[z_{b, \mathrm{eq}}\right]^{n_{b}},
$$

where $z_{i, \mathrm{eq}}=e^{\beta \mu_{i, \mathrm{eq}}^{\mathrm{v}} / \Lambda_{i}^{3}}$ is the fugacity of component $i$ in equilibrium vapor. It is important to note that the chemical potential of a molecule inside an arbitrary binary cluster is not the same as in the surrounding bulk equilibrium vapor-it depends on the cluster composition. We present $q_{\mathbf{n}}$ in the coarse-grained form,

$$
q_{\mathbf{n}}=\frac{1}{n_{a} !} \oint_{\mathrm{cl}} d \mathbf{R}^{n_{a}} e^{-\beta \mathcal{H}^{\mathrm{CG}}}
$$

in which the positions of $b$ particles are integrated out; $\mathcal{H}^{\mathrm{CG}}=U_{a a}\left(\left\{\mathbf{R}_{a}^{\left.n_{a}\right\}}\right)+\mathcal{F}_{b / a}\left(\left\{\mathbf{R}_{a}^{n_{a}}\right\} ; n_{a}, n_{b}, T\right)\right.$ is the Hamiltonian of the equivalent single-component cluster, where $\mathcal{F}_{b / a}\left(\left\{\mathbf{R}_{a}^{n_{a}}\right\}, n_{a}, n_{b}, T\right)=-k_{\mathrm{B}} T \ln q_{b / a}$ is the free energy of $b$ molecules in the instantaneous environment of $a$ molecules,

$$
q_{b / a}\left(\left\{\mathbf{R}_{a}^{\left.n_{a}\right\}}\right) \equiv \frac{1}{n_{b} !} \oint_{\mathrm{cl}} d \mathbf{r}^{n_{b}} e^{-\beta\left(U_{b b}+U_{a b}\right)} .\right.
$$

By construction $\mathcal{H}^{\mathrm{CG}}$ guarantees that $q_{\mathbf{n}}$ and hence all thermodynamic properties of the original $\left(n_{a}, n_{b}\right)$ cluster coincide with those of the single-component cluster. The diagrammatic expansion of $\ln q_{b / a}$ in the Mayer functions of $a-b$ and $b-b$ interactions results in the expansion of $\mathcal{F}_{b / a}$ in $m$-body effective interactions between $a$ molecules [10],

$$
\mathcal{F}_{b / a}=\mathcal{F}_{0}\left(n_{a}, n_{b}, T\right)+U_{2}\left(\left\{\mathbf{R}_{a}^{n_{a}}\right\} ; x_{b}, T\right)+\cdots,
$$

where $x_{b}=n_{b}^{1} /\left(n_{a}^{1}+n_{b}^{1}\right)$. The zeroth-order contribution $\mathcal{F}_{0}\left(n_{a}, n_{b}, T\right)$, called the volume term, does not depend on positions of molecules but is important for thermodynamics since it depends on cluster composition. Thus, $\mathcal{H}^{\mathrm{CG}}$ $=\mathcal{F}_{0}\left(n_{a}, n_{b}, T\right)+U^{\mathrm{CG}}\left(\left\{\mathbf{R}_{a}^{n_{a}}\right\} ; x_{b}, T\right)$ with the total coarsegrained interaction energy,

$$
U^{\mathrm{CG}}=U_{a a}\left(\left\{\mathbf{R}_{a}^{\left.n_{a}\right\}}\right)+U_{2}\left(\left\{\mathbf{R}_{a}^{n_{a}}\right\} ; x_{b}, T\right)+\cdots .\right.
$$

This yields the formally exact result

$$
q_{\mathbf{n}}=e^{-\beta \mathcal{F}_{0}} q_{n_{a}}^{\mathrm{CG}}, \quad \text { where } q_{n_{a}}^{\mathrm{CG}}=\frac{1}{n_{a} !} \oint_{\mathrm{cl}} d \mathbf{R}^{n_{a}} e^{-\beta U^{\mathrm{CG}}} .
$$

By tracing out the degrees of freedom of $b$ molecules, we are left with the single-component cluster of pseudo-a molecules 
with an unknown interaction potential $u^{\mathrm{CG}}\left(R ; x_{b}, T\right)$, which depends on $x_{b}$ and $T$.

The volume term is $\mathcal{F}_{0}=\mathcal{F}_{b, \text { id }}+\mathcal{F}_{0 \text {,exc }}$, where $\mathcal{F}_{b \text {,id }}$ $=k_{\mathrm{B}} T n_{b} f_{b, \text { id }}$ is the free energy of ideal gas of $b$ molecules in the cluster volume $V_{\mathrm{cl}}=\sum_{i} n_{i} v_{i}^{1}$, and $\mathcal{F}_{0 \text {,exc }}$ is the excess (over ideal) contribution due to $b-b$ and $a-b$ interactions; $f_{b \text {,id }}$ depends only on intensive quantities. The calculation of $\mathcal{F}_{0 \text {,exc }}$ in terms of interaction potentials is a very challenging task. For our purposes, however, we do not need to know its exact form but can make use of the general statement that $\mathcal{F}_{0 \text {,exc }}$ is a homogeneous function of the first order in $n_{a}$ and $n_{b}$ [14]: $\beta \mathcal{F}_{0, \text { exc }}=n_{b} f_{0, \text { exc }}$, with $f_{0 \text {,exc }}$ depending only on intensive quantities. Thus, the volume term becomes $\exp \left[-\beta \mathcal{F}_{0}\right]$ $=\Phi_{b}^{n_{b}}$, where $\Phi_{b}$ is an unknown function of $x_{b}$ and $T$. The configuration integral $q_{n_{a}}^{\mathrm{CG}}$ of the cluster with $n_{a}$ identical particles can be analyzed using the formalism of the singlecomponent MKNT of Ref. [8], in which an arbitrary $n$ cluster is considered to consist of the two groups of molecules: the core $n^{\text {core }}$ and the surface $n^{s}$, fluctuating around their mean values $\overline{n^{\text {core }}}(n)$ and $\overline{n^{s}}(n)$, and

$$
\frac{q_{n_{a}}^{\mathrm{CG}}}{V}=C \Phi_{a}^{n_{a}} e^{-\theta_{\text {micro }} \bar{n}_{a}^{s}}\left(n_{a}\right) .
$$

Here, $\Phi_{a}=1 / z_{\text {sat }}$, where $z_{\text {sat }}(T)$ is the fugacity at saturation; $\theta_{\text {micro }}$ is the reduced free energy per surface particle, termed in [8] the "reduced microscopic surface tension." MKNT allows one to express the parameters in Eq. (9) in terms of the equilibrium properties of the substance,

$$
\theta_{\text {micro }}=-\ln \left(-\frac{B_{2} p_{\text {sat }}}{k_{\mathrm{B}} T}\right), \quad C=\frac{p_{\text {sat }}}{k_{\mathrm{B}} T} e^{\theta_{\text {micro }}},
$$

where $B_{2}(T)$ is the second virial coefficient and $p_{\text {sat }}(T)$ is the saturation pressure; $\overline{n_{a}^{s}}$ depends parametrically on the coordination number in the liquid phase. Within the coarse-grained procedure, the properties of the pseudo- $a$ fluid are functionals of $u^{\mathrm{CG}}$. It is practically impossible to restore $u^{\mathrm{CG}}$ from the microscopic considerations. An alternative to the microscopic approach is the use of the known asymptotic features of $\varrho_{\text {eq }}\left(n_{a}, n_{b}\right)$. Substitution of Eqs. (8) and (9) into Eq. (6) yields

$$
\varrho_{\mathrm{eq}}\left(n_{a}, n_{b}\right)_{p^{\mathrm{v}}, T}=C\left(\prod_{i}\left[\Phi_{i}\left(x_{b}\right) z_{i, \mathrm{eq}}\right]^{n_{i}}\right) e^{-\theta_{\mathrm{micro}} \bar{n}_{a}^{s}}
$$

Intensive thermodynamic properties of the cluster molecules depend on the bulk composition $x_{b}$, implying that $\Phi_{a}, \Phi_{b}, \theta_{\text {micro }}$ must be considered functions of $x_{b}$. Let us fix $x_{b}$ and consider the two-phase equilibrium at $p^{\operatorname{coex}}\left(x_{b}, T\right)$, which is the pressure above the bulk binary solution with composition $x_{b}$. In this state the fugacities are $z_{i, \text { coex }}$ $=e^{\beta \mu_{i, \text { coex }}} / \Lambda_{i}^{3}$, where $\mu_{i, \text { coex }}\left(x_{b}\right)$ is the chemical potential at $x_{b}$ equilibrium. The distribution function for this state has the form of Eq. (11) where $z_{i, \text { eq }}$ is replaced with $z_{i, \text { coex }}$. Now, from the entire cluster size space let us consider the clusters falling on the $x_{b}$-equilibrium line $n_{b}^{1}=n_{a}^{1}\left(x_{b} / x_{a}\right)$. The Gibbs formation energy of such a cluster contains only the surface term, $\varrho\left(n_{a}, n_{b}\right)_{p^{\text {coex }}, T} \sim \exp \left[-\theta_{\text {micro }} \bar{n}_{a}^{s}\left(n_{a} ; x_{b}, T\right)\right]$, implying that $\Phi_{i}\left(x_{b}\right)=1 / z_{i, \text { coex }}\left(x_{b}\right)$. Now we can identify

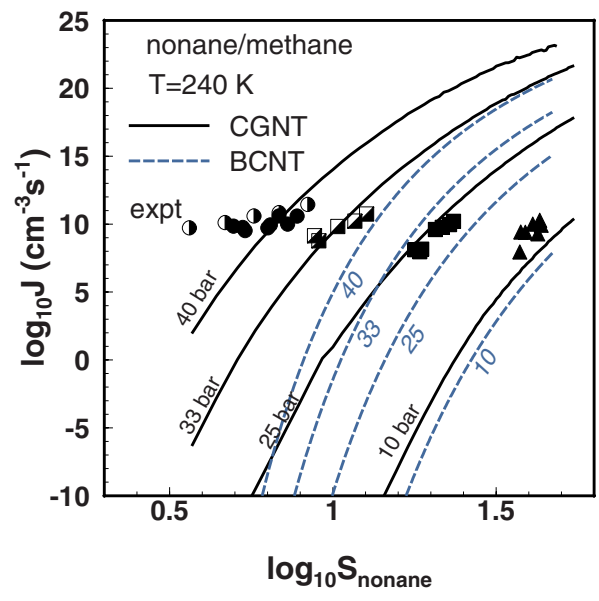

FIG. 1. (Color online) Nonane/methane nucleation at $T$ $=240$ K. Solid lines: CGNT; dashed lines: BCNT. Symbols: experiment $[4,16]$.

$$
p_{\text {sat }}=y_{a}^{\operatorname{coex}}\left(x_{b}\right) p^{\operatorname{coex}}\left(x_{b}\right),
$$

where $y_{i}^{\operatorname{coex}}\left(x_{b}\right)$ is the equilibrium vapor molar fraction of component $i$ at $x_{b}$ equilibrium. Since $u^{\mathrm{CG}}$ is not known, we have to introduce an ansatz for $B_{2}$, which should depend on $x_{b}$. The simplest form, satisfying the pure component limit, is $B_{2}=\sum_{i, j} x_{i} x_{j} B_{2, i j}$, where $B_{2, i i}(T)$ is the second virial coefficient of the pure component $i$, and $B_{2, a b}(T)$ is the cross virial term; the calculation of these quantities is discussed in [15]. Combining Eq. (11) with Eqs. (10) and (12), we obtain

$$
\begin{aligned}
\varrho_{\mathrm{eq}}\left(n_{a}, n_{b}\right)_{p^{\mathrm{v}}, T}= & {\left[\beta y_{a}^{\operatorname{coex}}\left(x_{b}\right) p^{\operatorname{coex}}\left(x_{b}\right)\right] e^{-g_{\mathrm{eq}}\left(n_{a}, n_{b} ; x_{b}\right)}, } \\
g_{\text {eq }}\left(n_{a}, n_{b} ; x_{b}\right)= & -\sum_{i} n_{i} \ln \left[\frac{y_{i, \mathrm{eq}} p^{\mathrm{v}}}{y_{i}^{\operatorname{coex}}\left(x_{b}\right) p^{\operatorname{coex}}\left(x_{b}\right)}\right] \\
& +\theta_{\text {micro }, a}\left(x_{b}\right)\left[\overline{n_{a}^{s}}\left(n_{a} ; x_{b}\right)-1\right] .
\end{aligned}
$$

The critical cluster is the saddle point of the free-energy surface $g\left(n_{a}, n_{b}\right)$, given by Eqs. (2) and (13), in the space of total numbers $n_{i}$. Note that the ambiguity in the prefactor $C$, inherent to BCNT, is eliminated.

Our choice to trace out the $b$ molecules in the cluster in favor of $a$ molecules could have been reversed: we could trace out $a$ molecules to be left with the effective Hamiltonian for the $b$ molecules resulting in Eq. (8) with the single-component cluster containing pseudo- $b$ particles. Although Eq. (8) is exact, the calculation of $q_{n_{a}}^{\mathrm{CG}}$ in it invokes approximations inherent to MKNT. Its domain of validity is [8] $\left|\beta B_{2} y_{i}^{\text {coex }} p^{\text {coex }}\right| \ll 1$, where $i=a$ or $b$. It is clear that to obtain accurate predictions one has to trace out the more volatile component (which we assumed to be component $b$ ).

We discuss implication of the proposed coarse-grained nucleation theory (CGNT) to nucleation of $n$-nonane $(a)$ and methane $(b)$ in the absence of carrier gas. The properties of the mixture at coexistence are found from the RedlichKwong-Soave equation of state [15]. If $p^{\mathrm{v}}$ is sufficiently high, methane not only removes the latent heat (thus, acting as a carrier gas) but also takes part in the nucleation process due to unlike interactions. Figure 1 shows CGNT nucleation 


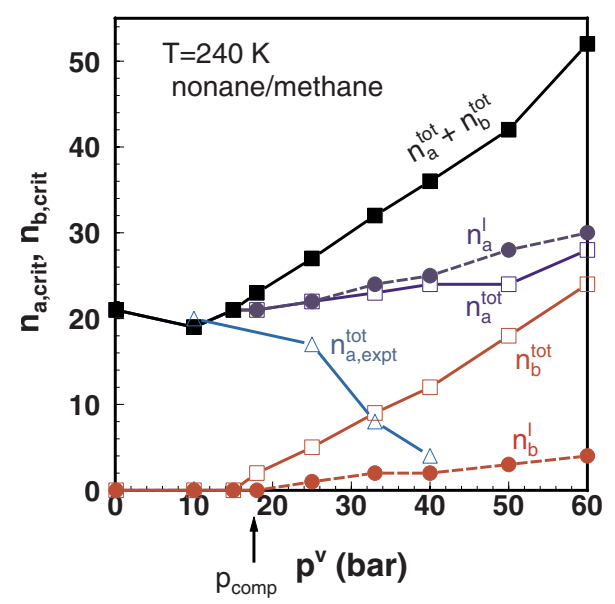

FIG. 2. (Color online) Critical cluster at fixed $J$ $=10^{10} \mathrm{~cm}^{-3} \mathrm{~s}^{-1}$ at $T=240 \mathrm{~K}$ as a function of $p^{\mathrm{v}} ; n_{i}^{\text {tot }} \equiv n_{i}=n_{i}^{1}$ $+n_{i}^{\text {exc }}$. Also shown is $n_{a \text {,expt }}^{\text {tot }}$ obtained from experimental data sets. The vertical arrow indicates $p_{\text {comp }}(T)$ [18].

rates for $T=240 \mathrm{~K}$ and various $p^{\mathrm{v}}$ together with experimental data of $[4,16]$. Also shown are the BCNT predictions of Ref. [17]. The agreement between CGNT and experiment lies within the range of experimental accuracy for most of the conditions except for extremely low $S_{a}<5$ at 40 bar. BCNT works fairly well for low $p^{\mathrm{v}}$ (10 and 25 bar) but fails drastically at higher $p^{\mathrm{v}}$. Predictions of the critical cluster content at a fixed $J=10^{10} \mathrm{~cm}^{-3} \mathrm{~s}^{-1}$ are shown in Fig. 2. At $p^{\mathrm{v}}<18$ bar nucleation can be viewed as a single-component process. Beyond 18 bar methane penetrates into the critical cluster; the nucleation process demonstrates binary features, becoming pronounced at higher $p^{\mathrm{v}}$. It is remarkable that this change occurs at the "compensation pressure" $p_{\text {comp }}[18]$ beyond which the partial molecular volume of nonane in the vapor phase becomes negative. As $p^{\mathrm{v}}$ is increased, $n_{a}^{\text {tot }}$ grows very slowly, while $n_{b}^{\text {tot }}$ increases rapidly. At high $p^{\mathrm{v}}$ the critical cluster is a nanosized object with a core-shell structure: its interior is rich in nonane while methane is predominantly adsorbed on the $K$ surface. From the slopes of experimental data sets in Fig. 1 one can determine, using nucleation theorem, $n_{a, \text { expt }}^{\text {tot }}$ corresponding to $J_{\text {expt }}=10^{10} \mathrm{~cm}^{-3} \mathrm{~s}^{-1}$. Figure 2 demonstrates different tendencies of experimental and theoretical critical cluster contents: $n_{a \text {,expt }}^{\text {tot }}$ falls with $p^{\mathrm{v}}$ while theoretical $n_{a}^{\text {tot }}$ slowly increases. This discrepancy results in the departure of CGNT curves from experiment at high $p^{\mathrm{v}}$.

In conclusion, the proposed model, which takes into account adsorption and treats small clusters without invoking capillarity approximation, opens a route toward studying nucleation in complex systems with nanosized critical clusters.

It is a pleasure to thank Howard Reiss and Gerry Wilemski for stimulating discussions. Dennis van Putten is acknowledged for performing saddle-point calculations using Laplacian smoothing [19].
[1] H. Reiss, J. Chem. Phys. 18, 840 (1950).

[2] D. Stauffer, J. Aerosol Sci. 7, 319 (1976).

[3] C. Flageollet et al., J. Chem. Phys. 72, 544 (1980); B. Wyslouzil et al., ibid. 94, 6827 (1991); R. Strey and Y. Viisanen, ibid. 99, 4693 (1993).

[4] C. C. M. Luijten et al., J. Chem. Phys. 111, 8535 (1999).

[5] H. Vehkamäki, Classical Nucleation Theory in Multicomponent Systems (Springer-Verlag, Berlin, 2006).

[6] G. Wilemski, J. Chem. Phys. 80, 1370 (1984).

[7] J. S. Rowlinson and B. Widom, Molecular Theory of Capillarity (Clarendon, Oxford, 1982).

[8] V. I. Kalikmanov, J. Chem. Phys. 124, 124505 (2006).

[9] S. Sinha et al., J. Chem. Phys. 132, 064304 (2010).

[10] C. N. Likos, Phys. Rep. 348, 267 (2001).
[11] J. L. Katz and H. Wiedersich, J. Colloid Interface Sci. 61, 351 (1977).

[12] A. Laaksonen et al., J. Chem. Phys. 111, 2019 (1999).

[13] Y. S. Djikaev et al., J. Chem. Phys. 120, 9752 (2004).

[14] V. I. Kalikmanov, Phys. Rev. E 68, 010101(R) (2003).

[15] R. C. Reid, J. M. Prausnitz, and B. E. Poling, The Properties of Gases and Liquids, 4th ed. (McGraw-Hill, New York, 1987).

[16] P. Peeters, Ph.D. thesis, Eindhoven University, 2002; D. G. Labetski, Ph.D. thesis, Eindhoven University, 2007.

[17] K. N. H. Looijmans et al., J. Chem. Phys. 102, 4531 (1995).

[18] V. I. Kalikmanov and D. G. Labetski, Phys. Rev. Lett. 98, 085701 (2007).

[19] F. O’Sullivan, J. Am. Stat. Assoc. 85, 213 (1990). 